\title{
Confusing Finding in Hepatic Injury With Diaphragmatic Rupture
}

\author{
Pil Young Jung \\ Department of Surgery, Yonsei university Wonju college of medicine, Wonju Severance Christian Hospital, Trauma center
}

Diaphragmatic injuries are relatively rare after blunt trauma. In some situations, these injuries may be hard to characterize during the initial survey and evaluation. In the case described here, diaphragmatic rupture with hepatic injury had confusing manifestations.

Key Words: Diaphragmatic injury, Hepatic injury

(Trauma Image Proced 2019(1):1-2)

\section{CASE}

A 34-year-old man who had no medical history was admitted via an emergency room with blunt trauma. At admission, he was in shock status, and focused abdominal sonography for trauma showed a minimal positive sign but no abdominal distention. A trauma series showed right-sided tension hemothorax, and a chest tube was inserted (Fig. 1.). More than $1000 \mathrm{~mL}$ gushed out within 30 minutes. Other injuries were fractures of the pelvic bone, tibia (open), and humerus. Therefore, emergency surgery for hemothorax was planned. Before going to surgery, I wanted to check the computed tomographic (CT) scan because the vital signs were stable for permissive hyportension. However, the CT scan showed a liver injury with unexpected diaphragmatic rupture (Fig. 2A., B.). Thus the plan for thoracic surgery had to be changed to abdominal surgery. A severe liver injury with diaphragmatic rupture

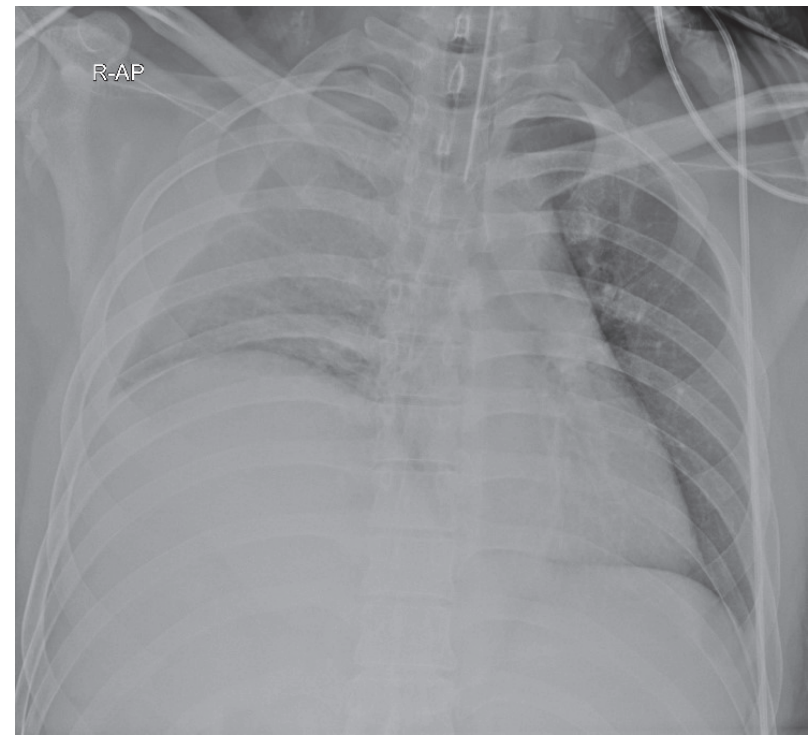

Fig. 1. Plain anteroposterior chest radiograph, showing rightsided tension hemothorax.

was found (Fig. 3.). Because of the diaphragmatic rupture, I had missed the presence of hemoperitoneum

Received: March 32019 Revised: May 8, 2019 Accepted: May 8, 2019

Correspondence to: Pil Young Jung, Department of Surgery, Yonsei university Wonju college of medicine, Wonju Severance Christian Hospital, Trauma center, 220-701, 20 Ilsan-ro, Wonju-si, Gangwon-do, South Korea

Tel: 82-33-741-0882, Fax: 82-33-741-0574, E-mail: surgery4trauma@yonsei.ac.kr

Copyright (c) 2019 Korean Association for Research, Procedures and Education on Trauma. All rights reserved.

(c) This is an open-access article distributed under the terms of the Creative Commons Attribution Non-Commercial License (http://creativecommons.org/ licenses/by-nc/4.0) which permits unrestricted noncommercial use, distribution, and reproduction in any medium, provided the original work is properly cited 

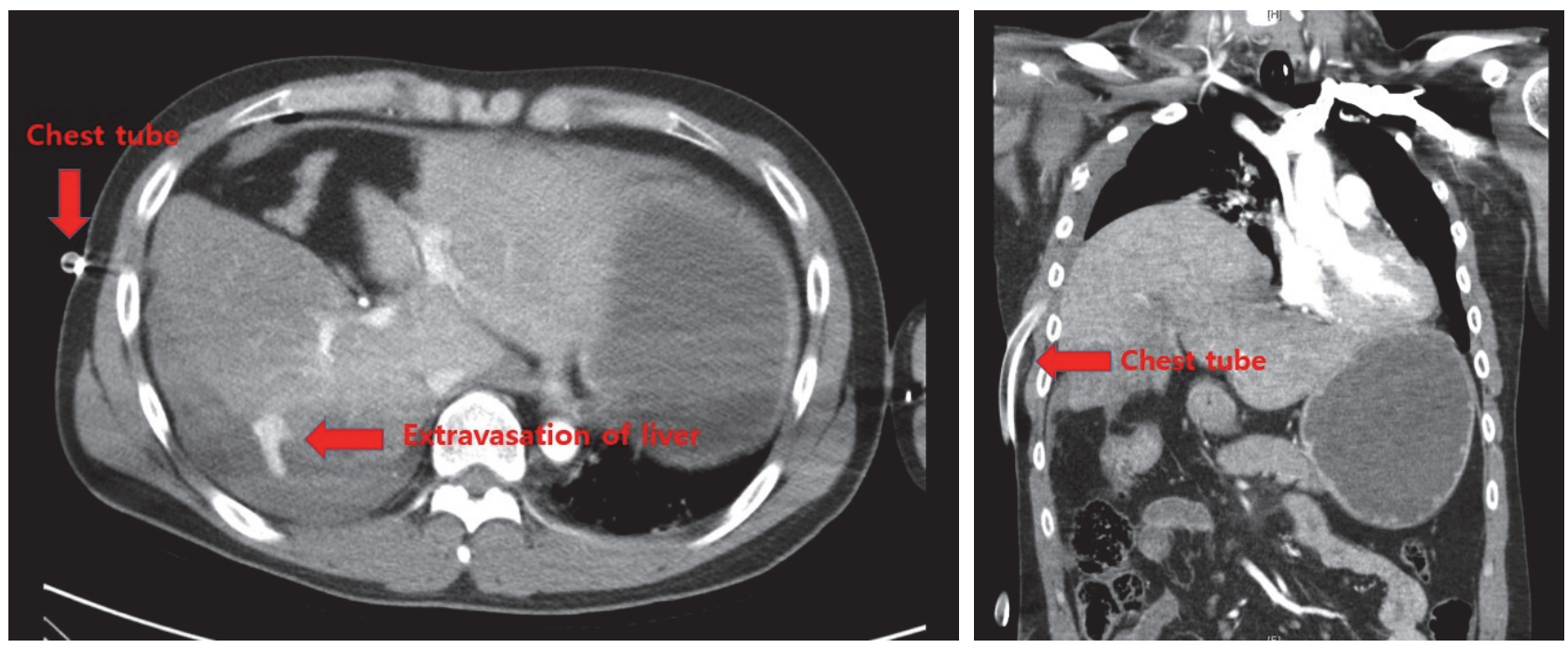

Fig. 2. Computed tomographic scan, showing diaphragmatic rupture with liver injury.

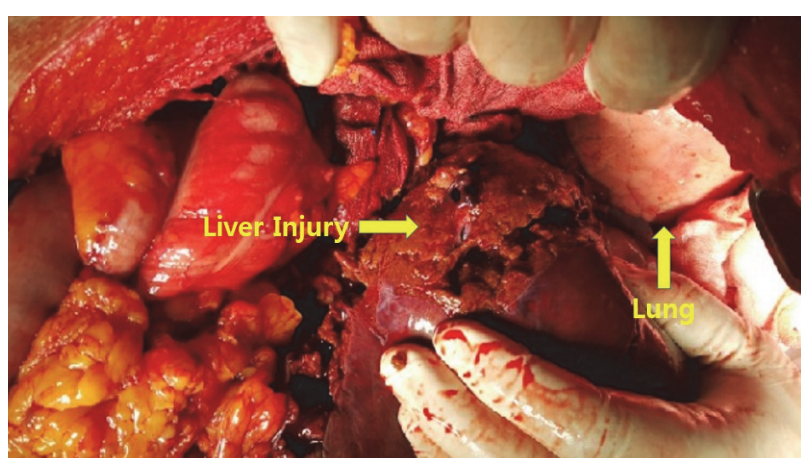

Fig. 3. Liver injury (grade IV) and diaphragmatic rupture in operation finding.

and misinterpreted the tension hemothorax as resulting from a chest injury. I performed laparotomy and midsternotomy and then attempted surgical damage control, including tape packing around the liver and temporary abdomen closure. However, the patient died 2 days after surgery.

\section{DISCUSSION}

Diaphragmatic injuries, including ruptures, are caused by thoracoabdominal blunt or penetrating trauma. They occur in a context of multiple traumas (1). Early diagnosis is most important, but evaluations of diaphragmatic rupture are not easy to find because diaphragmatic rupture often accompanies injuries to other organs, and intensive care, such as use of a ventilator, is necessary because vital signs are unstable (2). The diagnosis of diaphragmatic wounds and rupture remains difficult and is often delayed (3). In some trauma situations, proper diagnosis may be hard to establish during the initial survey and evaluation, and the manifestations may be confusing. Therefore, clinicians must use multidisciplinary approaches in treating multiple injuries.

\section{Conflicts of Interest Statement}

None of authors have a conflict of interest.

\section{REFERENCE}

1. Bosanquet D, Farboud A, Luckraz H (2009) A review diaphragmatic injury. Respir Med CME 2:1 - 6

2. Hwang SW, Kim HY, Byun JH. Management of patients with traumatic rupture of the diaphragm Korean $\mathrm{J}$ Thorac Cardiovasc Surg 2011 Oct;44(5):348-54

3. Thiam O, Konate I, Gueye ML et al. Traumatc diaphragmatic injuries: epidemiological, diagnostic and therapeutic aspects Springerplus 2016 Sep 20;5(1):1614 